\title{
NOTES
}

\section{New Journal}

Beginning in January 1965, Faraday Press inaugurated the bimonthly publication of Cybernetics, which is a cover-to-cover English translation of the Russian periodical entitled Kibernetika, published by the Academy of Sciences of the Ukrainian Soviet Socialist Republic. The editor-in-chief of the Russian periodical is V. M. Glushkov; the American editors and translators are not identified.

Published papers cover the following range of topics: general problems of ('ybernetics; theory of automata and algorithms; theory of digital and analog computers; algorithmic languages and theory of programming; methods of studying operations and systems; theory of optimum solutions; modeling of thinking processes; informational languages and systems; and mathematical linguistics.

The annual subscription is $\$ 115$; single issues are $\$ 35$ each.

Subscription orders and inquiries should be sent to The Faraday Press, Inc., 84 Fifth Avenue, New York, New York 10011.

\section{New Journal}

Springer-Verlag of Vienna and New York has announced the publication, beginning in early 1966, of a new international bilingual journal entitled Computing: Archives for Electronic Computing - Archiv für Elektronisches Rechnen, which is a continuation of the earlier Zeitschrift für Moderne Rechentechnik und Automation.

This journal will publish original papers and possibly reviews in German and English in the following fields: numerical applications of computers; nonnumerical applications of computers, such as language translation, learning processes, and information storage and retrieval; programming languages; operations research; and problems pertaining to information theory, automatic control, and data processing.

Publication will be at frequent, irregular intervals. One volume, consisting of four issues, will be issued each year, at a subscription rate of $\$ 24.00$.

The editors are: E. Bukovies, Vienna; R. Inziger, Vienna; W. Knödel, Stuttgart; and J. Menkes, Arlington.

Subscription orders and inquiries should be sent to Springer-Verlag New York, Inc., 175 Fifth Avenue, New York, New York 10010.

\section{New Journal}

Academic Press is proud to announce that it will soon be publishing the Journal of Computer and Systems Sciences. A new journal, to be issued quarterly, it will be devoted to the newly emerging interdisciplinary area of Computer and Systems Sciences. It will feature papers on computers and computer-like information proccssing systems; on the general theory of systems whether they be discrete (such as automata) or continuous, whether deterministic or stochastic, and including biological systems; and on optimization theory of such systems, whether for information systems or control systems, in which the emphasis is on computational methods and algorithms. The papers, largely theoretical in character with no compromise in mathematical rigor, will deal with problems that may arise in diverse 
disciplines but will involve the computer or computer-based technology in an essential way.

The editors of Journal of Computer and Systems Sciences will be Professor A. V. Balakrishnan, University of California, Los Angeles; Professor E. K. Blum, Wesleyan University; Dr. R. W. Hamming, Bell Telephone Laboratorics; Professor P. D. Lax, New York University; and Professor L. A. Zadeh, University of California, Berkeley. They will be assisted by an international editorial board representing the U.S.S.R., Israel, England, France, and Japan.

\section{New Journal}

Academic Press will soon publish a new journal, the Journal of Combinatorial. Theory, devoted to the publication of original mathematical articles dealing with theoretical and physical aspects of the study of finite and discrete structures in all branches of science.

The editors-in-chief of the new journal are Professor Marshall Hall, California Institute of Technology, Dr. Mark Kac, The Rockefeller Institute, Professor Claude Shannon, Massachusetts Institute of Technology, Professor W. T. Tutte, University of Waterloo, Dr. G. E. Uhlenbeck, The Rockefeller Institute, and Professor S. M. Ulam, University of Colorado.

Members of the editorial board are Dr. Claude Berge, International Computation Centre, Rome, Professors R. C. Bose, University of North Carolina, George B. Dantzig, University of California, Berkeley, N. G. de Bruijn, Technological University, Eindhoven, R. J. Duffin, Carnegie Institute of Technology, Freeman J. Dyson, Institute for Advanced Studies, Paul Erdös, The Hungarian Academy of Sciences, Dr. D. R. Fulkerson, RAND Corporation, Robert Gallager, Massachusetts Institute of Technology, J. M. H. Hammersley, University of Oxford, Frank Harary, University of Michigan, Dr. Alan J. Hoffman, IBM Research Center, Victor Klee, University of Washington, N. S. Mendelsohn, University of Manitoba, Leo Moser, University of Alberta, Oystein Ore, Yale University, Michael Rabin, Hebrew University of Jerusalem, Alfred Rényi, The Hungarian Academy of Sciences, Mr. John Riordan, Bell Telephone Laboratories, Gerhard Ringel, Freie Universität, Gian-Carlo Rota, The Rockefeller Institute, Herbert Ryser, Syracuse University, J. W. T. Youngs, University of California, Santa Cruz, A. A. Zykov, Academy of Sciences, Novosibirsk.

Manuscripts may be submitted to the editors-in-chief or to any member of the editorial board and will be accepted in English, French or German. Copies of "Information for Authors" are available from the publishers upon request.

Each author will receive fifty free reprints of his paper. Additional copies may be purchased.

The Journal of Combinatorial Theory will be issued quarterly. Volume 1, No. 1, is scheduled for publication in the spring of 1966 .

\section{An Announcement Concerning the Delay Line SIEVE DLS-127}

\section{By D. H. Lehmer}

This machine is now operational and a substantial percentage of its free time is now available to number theorists and combinatorial analysts everywhere. 
The DLS-127 combines up to 31 sets of arithmetical progressions, whose differences are primes or powers of primes less than or equal to 127, at the rate of a little over a million cases per second. It is thus available to solve the wide variety of problems to which this operation is basic.

A detailed description of the device will appear elsewhere.

An automatic set-up procedure has thus far been programmed only for the diophantine equation $f(x, y)=0$, where $f$ is a polynomial of arbitrary degree with coefficients that are integers of arbitrary size. To illustrate the generality of this program, we give the following list of problems that are special cases of solving $f(x, y)=0$.

(1) Find all the solutions $(x, y)$ with $x<L$ of the equation $x^{3}+D=y^{2}$ for a given value of $D$.

(2) Find the representations of a large number by a given binary quadratic form, for example $N=x^{2}-y^{2}$.

(3) Find the numbers (or the number of numbers) less than $L$ which are quadratic (or higher power) residues of each of a set of given primes $<127$.

(4) For a given polynomial $g$ find the number of numbers between given limits for which $g(x)$ is divisible by none of a given set of primes $<127$.

(5) Find the binomial units of a given algebraic number field.

(6) For a given polynomial $g$ find the numbers (or the number of numbers) between given limits that are represented by $g$.

Should the user have a problem which can be put into the form $f(x, y)=0$ he needs to submit only the coefficients of $f$. For other sieve problems special input decks must be prepared at present. Since the search for $x$ proceeds at the rate of a million per second, the SIEVE can process $10^{11}$ integers in a reasonable time. Although such problems can be coded for an all-purpose computer, they run on the special purpose sieve at speeds that are higher by one or two orders of magnitude.

Problems may be submitted, and inquiries made, by writing to

\section{SIEVE}

Department of Mathematics

University of California

Berkeley, California 94704

\section{Newsletter for Numerical Analysts}

The ACM Special Interest Committee on Numerical Mathematics (SICNUM) will begin publication of a newsletter in order to provide numerical analysts with a fast means of communication below the journal level. The newsletter is free and will be sent upon request. The newsletter will appear as frequently as there is sufficient material.

Requests to receive the newsletter, material for the newsletter, and requests for additional information should be sent to the chairman of SICNUM,

Dr. J. F. Traub

Bell Telephone Laboratories, Inc.

Murray Hill, New Jersey 